\title{
Some constancy results for nematic liquid crystals and harmonic maps
}

\author{
by \\ Kai Seng CHOU \\ Department of Mathematics, \\ The Chinese University of Hong Kong, \\ Shatin, Hong Kong. \\ and \\ Xi-Ping ZHU \\ Department of Mathematics, \\ Zhongshan University, \\ Guangzhou, P. R. China.
}

ABSTRACT. - Uniqueness results for nematic liquid crystals and harmonic maps with constant boundary data are established. Nonsolvability on non-starshaped domains for a semilinear elliptic equation is also given.

Key words: Nematic liquid crystals, harmonic maps, Pohozaev identity, non-star shaped domains.

RÉSUMÉ. - Des résultats d'unicité pour les cristaux liquides nématiques et pour les applications harmoniques avec des conditions aux bords constantes sont établis. Un résultat de non-existence pour une équation semilinéaire elliptique dans des domaines qui ne sont pas étalés est également présenté.

Classification A.M.S.: $35 \mathrm{~J} 60$.

Annales de l'Institut Henri Poincaré - Analyse non linéaire - 0294-1449

Vol. 12/95/01/\$4.00/

(C) 1995 L'Association Publications de l'Institut Henri Poincaré. Published by Elsevier B.V. All rights reserved 


\section{INTRODUCTION}

A liquid crystal is a mesomorphic phase of a material which occurs between its liquid and solid phases. Frequently, molecules comprising the material are relatively rigid and rod-like. The equilibrium configuration of the liquid crystal may be described in terms of its optical axes, a unit vector field $n$ defined on the region $\Omega$ in $\mathbb{R}^{3}$ occupied by the material. For a nematic liquid crystal, the Oseen-Frank free energy density $W$ is given by

$$
\begin{aligned}
W(n, \nabla n)= & k_{1}(\operatorname{div} n)^{2}+k_{2}(n \cdot \operatorname{curl} n)^{2}+k_{3}|n \wedge \operatorname{curl} n|^{2} \\
& +\left(k_{2}+k_{4}\right)\left[\operatorname{tr}(\nabla n)^{2}-(\operatorname{div} n)^{2}\right]
\end{aligned}
$$

where the $k_{i}$ are material constants, $k_{i}>0$ for $i=1,2,3$ and $k_{2} \geq\left|k_{4}\right|$. In the special case $k_{1}=k_{2}=k_{3}=1$ and $k_{4}=0$,

$$
W(n, \nabla n)=|\nabla n|^{2}
$$

which is the integrand for a harmonic mapping from a domain $\Omega$ in $\mathbb{R}^{3}$ into $S^{2}$.

Let us set

$$
E(n)=\int_{\Omega} W(n, \nabla n) d x .
$$

In this paper we shall study the following question: Suppose $n$ is a stationary point for the bulk energy $E(n)$ which is equal to a constant on the boundary of $\Omega$. Is it necessary that $n$ becomes a constant throughout $\Omega$ ? Note that $n$ being constant is always a solution of the Euler-Lagrange equation associated with the bulk energy. So a constancy result is in fact a uniqueness theorem for the solution of a Dirichlet problem.

To our knowledge this question has not been studied in general before. But there are results for the special case (1.2). For instance, Lemaire [9], Wood [13], Karcher and Wood [8] established constancy results for smooth harmonic maps from an $n$-dimensional bounded, star-shaped domain (into a general target manifold). On the other hand, R. T. Smith constructed nonconstant harmonic maps from an annulus to the 2-sphere with constant boundary data. See Section $11.7(c)$ in [14]. Other related results can be found in [3]. So constancy results do not always hold. 
In this paper, we shall obtain a Pohozaev identity (cf. [11] and [12]) for smooth stationary points of the liquid crystal bulk energy (1.3). As a consequence of this identity, we show that no non-constant smooth stationary points can exist in a star-shaped domain $\Omega$ having prescribed constant boundary values (Theorem 1 ). Next, we take into account the effects of magnetic fields and get a similar constancy result (Theorem 2). We also study Ericksen's model [10] on nematic liquid crystals with variable degree of orientation, and deduce a constancy result (Theorem 3).

So far constancy results have been known on balls or star-shaped domains. A similar situation holds in the study of the semilinear Dirichlet problem: for $\Omega$ in $\mathbb{R}^{n}, n \geq 3$,

$$
\left\{\begin{array}{l}
\Delta u+u^{p}=0, \quad u>0, \quad \text { in } \Omega, \\
u=0 \text { on } \partial \Omega .
\end{array}\right.
$$

When $p \geq(n+2) /(n-2)$, Pohozaev's identity [11] implies that no positive solution can exist when $\Omega$ is a bounded, star-shaped domain. However, when $p=(n+2) /(n-2)$ and $\Omega$ has certain non-trivial topology, Bahri and Coron [1] showed that (1.4) admits a positive solution. On the other hand, Ding [4] has recently produced a contractible $\Omega$ over which (1.4) is solvable for $p=(n+2) /(n-2)$. In the final section of this work we shall construct a non-starshaped, ball-like domain over which (1.4) is not solvable for $p \geq(n+2) /(n-2)$. A constancy result for harmonic maps over this domain will also be obtained.

Throughout this paper, we use summation convention where repeated indices are understood to be summed from 1 to 3 .

\section{CONSTANCY RESULTS ON A STAR-SHAPED DOMAIN}

Let $\Omega \subset \mathbb{R}^{3}$ be a bounded domain with smooth boundary $\partial \Omega$, and let $\nu=\left(\nu^{1}, \nu^{2}, \nu^{3}\right)$ be the outer normal at $\partial \Omega$. Recall that $\Omega$ is called starshaped if there exists a point $x_{0} \in \Omega$ such that the line segment $\bar{x}_{0}$ is contained in $\Omega$ for all $x \in \Omega$.

Set

$$
H^{1}\left(\Omega ; S^{2}\right)=\left\{n \in H^{1}\left(\Omega ; \mathbb{R}^{3}\right):|n(x)|=1 \text { a.e. }\right\}
$$

and

$$
H_{n_{0}}^{1}\left(\Omega, S^{2}\right)=\left\{n \in H^{1}\left(\Omega ; S^{2}\right): n=n_{0} \text { on } \partial \Omega\right\},
$$

where $n_{0}: \partial \Omega \rightarrow S^{2}$ is the boundary datum.

Vol. $12, \mathrm{n}^{\circ} 1-1995$. 


\section{Oseen-Frank's model}

For a nematic liquid crystal, the Oseen-Frank free energy density $W$ is given by (cf. [6]).

$$
\begin{aligned}
W(n, \nabla n)= & k_{1}(\operatorname{div} n)^{2}+k_{2}(n \cdot \operatorname{curl} n)^{2}+k_{3}|n \wedge \operatorname{curl} n|^{2} \\
& +\left(k_{2}+k_{4}\right)\left[\operatorname{tr}(\nabla n)^{2}-(\operatorname{div} n)^{2}\right],
\end{aligned}
$$

where $k_{i}>0, i=1,2,3, k_{2} \geq\left|k_{4}\right|$, and $n \in H^{1}\left(\Omega ; S^{2}\right)$. As Oseen and Erickson [5] have observed, the last term is a surface energy density which does not contribute to the equilibrium system. In fact, by [7],

$$
\int_{\Omega}\left[\operatorname{tr}(\nabla n)^{2}-(\operatorname{div} n)^{2}\right] d x=\int_{\partial \Omega}\left[\left(\nabla_{T} n\right) n-\operatorname{tr}\left(\nabla_{T} n\right) n\right] \cdot \nu d \mathcal{H}^{2} .
$$

Thoughout this paper, we always choose

$$
W(n, \nabla n)=k_{\mathbf{1}}(\operatorname{div} n)^{2}+k_{2}(n \cdot \operatorname{curl} n)^{2}+k_{3}|n \wedge \operatorname{curl} n|^{2} .
$$

The bulk energy is

$$
E(n)=\int_{\Omega} W(n, \nabla n) d x, \quad \text { for } \quad n \in H^{1}\left(\Omega ; S^{2}\right) .
$$

We consider a stationary point $n$ of $(2.2)$ in $H_{n_{0}}^{1}\left(\Omega ; S^{2}\right)$,

$$
\left.\frac{d}{d t}\right|_{t=0} \int_{\Omega} W\left(\frac{n+t \zeta}{|n+t \zeta|}, \nabla\left(\frac{n+t \zeta}{|n+t \zeta|}\right)\right) d x=0
$$

for $\zeta \in H_{0}^{1}\left(\Omega ; \mathbb{R}^{3}\right) \cap L^{\infty}$. Then

$$
\begin{aligned}
0= & \left.\int_{\Omega} W_{p_{i}^{k}} \frac{d}{d t}\right|_{t=0} \frac{\partial}{\partial x_{i}}\left(\frac{n^{k}+t \zeta^{k}}{|n+t \zeta|}\right) d x \\
& +\left.\int_{\Omega} W_{u^{k}} \frac{d}{d t}\right|_{t=0}\left(\frac{n^{k}+t \zeta^{k}}{|n+t \zeta|}\right) d x \\
= & \int_{\Omega}\left\{W_{p_{i}^{k}}\left[\frac{\partial \zeta^{k}}{\partial x_{i}}-\frac{\partial}{\partial x_{i}}\left(n^{k} n^{j} \zeta^{j}\right)\right]+W_{u^{k}}\left(\zeta^{k}-n^{k} n^{j} \zeta^{j}\right)\right\} d x
\end{aligned}
$$

Thus the Euler-Lagrange equation for a stationary point of (2.2) is

$$
\left\{\begin{array}{c}
-\frac{\partial}{\partial x_{i}} W_{p_{i}^{k}}+n^{k} n^{j} \frac{\partial}{\partial x_{i}} W_{p_{i}^{j}}+W_{u^{k}}-n^{k} n^{i} W_{u^{i}}=0, \\
k=1,2,3
\end{array}\right.
$$

in $H^{-1}\left(\Omega ; \mathbb{R}^{3}\right)$. 
Now, let's assume the stationary point $n$ of (2.2) is smooth on $\bar{\Omega}$ and is equal to constant on $\partial \Omega$. For any $\bar{x} \in \Omega$, we have, by (2.3),

$$
\begin{aligned}
\frac{\partial}{\partial x_{i}} & {\left[\left(x_{i}-\bar{x}_{i}\right) W-\left(x_{j}-\bar{x}_{j}\right) \frac{\partial n^{k}}{\partial x_{j}} W_{p_{i}^{k}}\right] } \\
= & 3 W+\left(x_{i}-\bar{x}_{i}\right) \frac{\partial^{2} n^{k}}{\partial x_{i} \partial x_{j}} W_{p_{j}^{k}}+\left(x_{i}-\bar{x}_{i}\right) \frac{\partial n^{k}}{\partial x_{i}} W_{u^{k}} \\
& -\frac{\partial n^{k}}{\partial x_{i}} W_{p_{i}^{k}}-\left(x_{j}-\bar{x}_{j}\right) \frac{\partial^{2} n^{k}}{\partial x_{i} \partial x_{j}} W_{p_{i}^{k}}-\left(x_{j}-\bar{x}_{j}\right) \frac{\partial n^{k}}{\partial x_{j}} \frac{\partial}{\partial x_{i}} W_{p_{i}^{k}} \\
= & 3 W+\left(x_{i}-\bar{x}_{i}\right) \frac{\partial n^{k}}{\partial x_{i}} W_{u^{k}}-\frac{\partial n^{k}}{\partial x_{i}} W_{p_{i}^{k}}-\left(x_{j}-\bar{x}_{j}\right) \frac{\partial n^{k}}{\partial x_{j}} \frac{\partial}{\partial x_{i}} W_{p_{i}^{k}} \\
= & 3 W+\left(x_{i}-\bar{x}_{i}\right) \frac{\partial n^{k}}{\partial x_{i}} W_{u^{k}}-\frac{\partial n^{k}}{\partial x_{i}} W_{p_{i}^{k}} \\
& -\left(x_{j}-\bar{x}_{j}\right) \frac{\partial n^{k}}{\partial x_{j}}\left[\left(n^{k} n^{l} \frac{\partial}{\partial x_{i}} W_{p_{i}^{l}}\right)+W_{u^{k}}-n^{k} n^{l} W_{u_{l}}\right] \\
= & 3 W-\frac{\partial n^{k}}{\partial x_{i}} W_{p_{i}^{k}}-\left(x_{j}-\bar{x}_{j}\right) \frac{\partial n^{k}}{\partial x_{j}} n^{k} n^{l}\left(\frac{\partial}{\partial x_{i}} W_{p_{i}^{l}}-W_{u^{l}}\right)
\end{aligned}
$$

in $\bar{\Omega}$. Since $|n|=1$,

$$
n^{k} \frac{\partial n^{k}}{\partial x_{j}}=0 \text { for } j=1,2,3, \quad \text { in } \bar{\Omega}
$$

Therefore,

$$
\begin{aligned}
& \frac{\partial}{\partial x_{i}}\left[\left(x_{i}-\bar{x}_{i}\right) W-\left(x_{j}-\bar{x}_{j}\right) \frac{\partial n^{k}}{\partial x_{j}} W_{p_{i}^{k}}\right] \\
& =3 W-\frac{\partial n^{k}}{\partial x_{i}} W_{p_{i}^{k}} \quad \text { in } \bar{\Omega} .
\end{aligned}
$$

We integrate this identity over the domain $\Omega$. It follows from the divergence theorem that

$$
\begin{aligned}
& \int_{\partial \Omega}\left[(x-\bar{x}) \cdot \nu W-\left(x_{j}-\bar{x}_{j}\right) \frac{\partial n^{k}}{\partial x_{j}} W_{p_{i}^{k}} \nu^{i}\right] d \mathcal{H}^{2} \\
& =\int_{\Omega}\left(3 W-\frac{\partial n^{k}}{\partial x_{j}} W_{p_{j}^{k}}\right) d x
\end{aligned}
$$


Using the condition $n=$ const. on $\partial \Omega$, we have, for $i, k=1,2,3$,

$$
\frac{\partial n^{k}}{\partial x_{i}}=\frac{\partial n^{k}}{\partial \nu} \nu^{i} \quad \text { on } \partial \Omega
$$

So

$$
\left(x_{j}-\bar{x}_{j}\right) \frac{\partial n^{k}}{\partial x_{j}} W_{p_{i}^{k}} \nu^{i}=\frac{\partial n^{k}}{\partial x_{i}} W_{p_{i}^{k}}\left(x_{j}-\bar{x}_{j}\right) \nu^{j} \quad \text { on } \partial \Omega .
$$

Thus (2.5) becomes

$$
\begin{gathered}
\int_{\partial \Omega}(x-\bar{x}) \cdot \nu\left(W-\frac{\partial n^{k}}{\partial x_{i}} W_{p_{i}^{k}}\right) d \mathcal{H}^{2} \\
=\int_{\Omega}\left(3 W-\frac{\partial n^{k}}{\partial x_{i}} W_{p_{i}^{k}}\right) d x
\end{gathered}
$$

By quadratic dependence of $W$ on $p$ we can use Euler's identity $p_{i}^{k} W_{p_{i}^{k}}=2 W$ on the both sides of (2.7) yielding the following Pohozaev's identity:

$$
-\int_{\partial \Omega}(x-\bar{x}) \cdot \nu W d \mathcal{H}^{2}=\int_{\Omega} W d x .
$$

It leads to the following constancy result directly.

THEOREM 1. - Assume that $\Omega$ is star-shaped. Then any smooth stationary point of (2.2) which is constant on $\partial \Omega$ is constant in $\Omega$.

Proof. - We have

$$
\begin{aligned}
\int_{\Omega} W d x= & \int_{\Omega}\left\{k_{1}(\operatorname{div} n)^{2}+k_{2}(n \cdot \operatorname{curl} n)^{2}\right. \\
& \left.\quad+k_{3}|n \wedge \operatorname{curl} n|^{2}+k_{1}\left[\operatorname{tr}(\nabla n)^{2}-(\operatorname{div} n)^{2}\right]\right\} d x \\
& \geq \min \left\{k_{1}, k_{2}, k_{3}\right\} \int_{\Omega}\left(\operatorname{tr}(\nabla n)^{2}+|\operatorname{curl} n|^{2}\right) d x \\
& =\min \left\{k_{1}, k_{2}, k_{3}\right\} \int_{\Omega}|\nabla n|^{2} d x .
\end{aligned}
$$

On the left hand side of (2.8), since $\Omega$ is star-shaped with respect to some point $\bar{x}$ in $\Omega$, we have $(x-\bar{x}) \cdot \nu \geq 0$. Therefore,

$$
-\int_{\partial \Omega}(x-\bar{x}) \cdot \nu W d \mathcal{H}^{2} \leq 0
$$

So it follows at once that $n \equiv$ const. in $\Omega$. 


\section{Effects of magnetic fields}

For a given smooth divergence free magnetic field $H$, which is usually a constant, the magnetization vector is given by $M=\left(\chi_{0} I+\chi_{a} n \otimes n\right) H$, where $\chi_{0}$ and $\chi_{a}$ are real numbers ( $c f$. [6]). The energy contributed to the system is $F(n)=M \cdot H=\left\langle\chi_{0} H, H\right\rangle+\chi_{a}(\langle n, H\rangle)^{2}$ and the energy density in the magnetic field is

$$
\mathcal{F}(n, \nabla n)=W(n, \nabla n)+F(n)
$$

where $W(n, \nabla n)$ is the free energy density given in (2.1) for nematic liquid crystals.

Since the term $\left\langle\chi_{0} H, H\right\rangle$ in $F(n)$ is irrelevant in the energy, without loss of generality we may assume $\chi_{0}=0$. The total energy for (2.9) is

$$
\mathcal{F}(n)=\int_{\Omega}(W(n, \nabla n)+F(n)) d x, \quad \text { for } n \in H_{n_{0}}^{1}\left(\Omega ; S^{2}\right)
$$

Let $n$ be a stationary point of $(2.10)$ in $H_{n_{0}}^{1}\left(\Omega, S^{2}\right)$, that is,

$$
\begin{gathered}
0=\left.\frac{d}{d t}\right|_{t=0} \int_{\Omega}(W+F)\left(\frac{n+t \zeta}{|n+t \zeta|}, \nabla\left(\frac{n+t \zeta}{|n+t \zeta|}\right)\right) d x \\
=\int_{\Omega}\left\{W_{p_{i}^{k}}\left[\frac{\partial \zeta^{k}}{\partial x_{i}}-\frac{\partial}{\partial x_{i}}\left(n^{k} n^{j} \zeta^{j}\right)\right]+W_{u^{k}}\left(\zeta^{k}-n^{k} n^{j} \zeta^{j}\right)\right. \\
\left.\quad+F_{u^{k}}\left(\zeta^{k}-n^{k} n^{j} \zeta^{j}\right)\right\} d x
\end{gathered}
$$

for $\zeta \in H_{0}^{1}\left(\Omega ; \mathbb{R}^{3}\right) \cap L^{\infty}$. Hence the Euler-Lagrange equation for $(2.10)$ is

$$
\begin{aligned}
& -\frac{\partial}{\partial x_{i}} W_{p_{i}^{k}}+n^{k} n^{j} \frac{\partial}{\partial x_{i}} W_{p_{i}^{j}}+W_{u^{k}}-n^{k} n^{j} W_{u^{j}} \\
& +F_{u^{k}}-F_{u^{j}} n^{k} n^{j}=0
\end{aligned}
$$

$k=1,2,3$, in $H^{-1}\left(\Omega ; \mathbb{R}^{3}\right)$. From now on, we assume $H=\left(H^{1}, H^{2}, H^{3}\right)$ is a constant vector in $\Omega$.

Proposition 1. - If $n$ is a constant solution of (2.11), then either $H \| n$ or $H^{\perp} n$. 
Proof. - Since

$$
\begin{aligned}
F & =\chi_{a}\left(n^{i} H^{i}\right)^{2}, \\
F_{u^{k}} & =2 \chi_{a}\left(n^{i} H^{i}\right) H^{k}, \quad \text { and } \\
n^{k} n^{j} F_{u^{j}} & =2 \chi_{a}\left(n^{i} H^{i}\right)^{2} n^{k},
\end{aligned}
$$

it follows from the above Euler-Lagrange equations that either $H^{k}=$ $(H \cdot n) n^{k}$, i.e., $H \| n$, or $H^{\perp} n$.

Now let's assume that $n$ is a stationary point of (2.10) which is smooth on $\bar{\Omega}$ and $n=n_{0} \equiv$ Const. on $\partial \Omega$ satisfying $H^{\perp} n_{0}$ or $H \| n_{0}$ on $\partial \Omega$. For any fixed $\bar{x} \in \Omega$,

$$
\begin{aligned}
\frac{\partial}{\partial x_{i}}[ & \left.\left(x_{i}-\bar{x}_{i}\right)\left(\mathcal{F}-\chi_{a}\left(n_{0} \cdot H\right)^{2}\right)-\left(x_{j}-\bar{x}_{j}\right) \frac{\partial n^{k}}{\partial x_{j}} \mathcal{F}_{p_{i}^{k}}\right] \\
= & 3\left(W+F-\chi_{a}\left(n_{0} \cdot H\right)^{2}\right)+\left(x_{i}-\bar{x}_{i}\right) W_{p_{j}^{k}} \frac{\partial^{2} n^{k}}{\partial x_{i} \partial x_{j}} \\
& +\left(x_{i}-\bar{x}_{i}\right) W_{u^{k}} \frac{\partial n^{k}}{\partial x_{i}}+\left(x_{i}-\bar{x}_{i}\right) F_{u^{k}} \frac{\partial n^{k}}{\partial x_{i}}-\frac{\partial n^{k}}{\partial x_{i}} W_{p_{i}^{k}} \\
& -\left(x_{j}-\bar{x}_{j}\right) W_{p_{i}^{k}} \frac{\partial^{2} n^{k}}{\partial x_{i} \partial x_{j}}-\left(x_{j}-\bar{x}_{j}\right) \frac{\partial n^{k}}{\partial x_{j}} \frac{\partial}{\partial x_{i}} W_{p_{i}^{k}},
\end{aligned}
$$

which, by (2.11) and Euler's identity,

$$
\begin{aligned}
= & 3\left[W+F-\chi_{a}(n \cdot H)^{2}\right]+\left(x_{i}-\bar{x}_{i}\right) W_{u^{k}} \frac{\partial n^{k}}{\partial x_{i}} \\
+ & \left(x_{i}-\bar{x}_{i}\right) F_{u^{k}} \frac{\partial n^{k}}{\partial x_{i}}-2 W-\left(x_{j}-\tilde{x}_{j}\right) \frac{\partial n^{k}}{\partial x_{j}} \\
& \times\left(n^{k} n^{l} \frac{\partial}{\partial x_{i}} W_{p_{i}^{l}}+W_{u^{k}}-n^{k} n^{j} W_{u^{j}}+F_{u^{k}}-F_{u^{l}} n^{k} n^{l}\right)
\end{aligned}
$$

Then, by the fact that $|n|=1$,

$$
\begin{aligned}
& \frac{\partial}{\partial x_{i}}\left[\left(x_{i}-\bar{x}_{i}\right)\left(W+F-\chi_{a}\left(n_{0} \cdot H\right)^{2}\right)-\left(x_{j}-\bar{x}_{j}\right) \frac{\partial n^{k}}{\partial x_{j}} W_{p_{i}^{k}}\right] \\
& \quad=W+3\left(F-\chi_{a}\left(n_{0} \cdot H\right)^{2}\right) .
\end{aligned}
$$

As above, since $F-\chi_{a}\left(n_{0} \cdot H\right)^{2}=0$ on $\partial \Omega$ we get

$$
\begin{aligned}
\int_{\partial \Omega} & {\left[(x-\bar{x}) \cdot \nu W-\left(x_{j}-\bar{x}_{j}\right) \frac{\partial n^{k}}{\partial x_{j}} W_{p_{i}^{k}} \nu^{i}\right] d \mathcal{H}^{2} } \\
= & \int_{\Omega}\left[W+3\left(F-\chi_{a}\left(n_{0} \cdot H\right)^{2}\right)\right] d x
\end{aligned}
$$


Since $n=n_{0} \equiv$ const. on $\partial \Omega$, we deduce the following Pohozaev's identity as before:

$$
-\int_{\partial \Omega}(x-\bar{x}) \cdot \nu W d \mathcal{H}^{2}=\int_{\Omega}\left[W+3\left(F-\chi_{a}\left(n_{0} \cdot H\right)^{2}\right)\right] d x .
$$

THEOREM 2. - Assume that $\Omega$ is star-shaped and $n$ is a smooth stationary point of (2.10) with $\left.n\right|_{\partial \Omega}=n_{0} \equiv$ const. Suppose also that either $\chi_{a} \geq 0$ and $H^{\perp} n_{0}$ on $\partial \Omega$, or $\chi_{a} \leq 0$ and $H \| n_{0}$ on $\partial \Omega$. Then $n$ is constant in $\Omega$.

Proof. - From the proof in Theorem 1 we know

$$
\int_{\Omega} W d x \geq \min \left\{k_{1}, k_{2}, k_{3}\right\} \int_{\Omega}|\nabla n|^{2} d x .
$$

And, in the case $\chi_{a} \geq 0$ and $H^{\perp} n_{0}$ on $\partial \Omega$, we have

$$
\int_{\Omega} 3\left[F-\chi_{a}\left(n_{0} \cdot H\right)^{2}\right] d x=3 \chi_{a} \int_{\Omega}(n \cdot H)^{2} d x \geq 0 ;
$$

In the case $\chi_{a} \leq 0$, and $H \| n_{0}$ on $\partial \Omega$, we have

$$
\int_{\Omega} 3\left(F-\chi_{a}\left(n_{0} \cdot H\right)^{2}\right) d x=3 \int_{\Omega}\left|\chi_{a}\right|\left[|H|^{2}-(n \cdot H)^{2}\right] d x \geq 0 .
$$

On the left hand of (2.13), we fix $\bar{x}$ such that $\Omega$ is star-shaped with respect to $\bar{x} \in \Omega$. Then

$$
-\int_{\partial \Omega}(x-\bar{x}) \cdot \nu W d \mathcal{H}^{2} \leq 0 .
$$

So it follows at once that $n \equiv$ const. in $\Omega$.

\section{Erickson's model}

In Erickson's model [10], the equilibrium configuration of a liquid crystal is described by a pair, $(s, n)$, where $s: \Omega \rightarrow\left[-\frac{1}{2}, 1\right]$ is a real-valued function which represents the variable degree of orientation of the liquid crystal and $n: \Omega \rightarrow S^{2}$ denotes the axes of otpical directions. It is a stationary point of the following bulk-energy functional in $H^{1}\left(\Omega ;\left[-\frac{1}{2}, 1\right] \times S^{2}\right)$ :

(2.14) $\int_{\Omega} \tilde{W}(s, \nabla s, n, \nabla n) d x$

$$
=\int_{\Omega}\left(W_{0}(s)+s^{2} W(n, \nabla n)+k_{5}|\nabla s|^{2}+k_{6}|\nabla s \cdot n|^{2}\right) d x,
$$


where $k_{5}, k_{6}>0, W(n, \nabla n)$ is the free energy density $(2.1)$, and $W_{0}(s)$ is a positive $C^{2}$ functions in $\left(-\frac{1}{2}, 1\right)$ satisfying

$$
\begin{aligned}
& W_{0}\left(-\frac{1}{2}\right)=W_{0}(1)=+\infty \\
& W_{0}\left(s^{*}\right)=\min _{s \in[-1 / 2,1]} W_{0}(s), \quad s^{*}>0, \text { and } W_{0}^{\prime}(0)=0 .
\end{aligned}
$$

Let's assume the stationary points $(s, n)$ is smooth in $\bar{\Omega}$. As above the pair $(s, n)$ satisfies the following Euler-Lagrange equation of (2.14). According to

$$
\begin{gathered}
0=\left.\frac{d}{d t}\right|_{t=0} \int_{\Omega} \tilde{W}\left(s+t \eta, \nabla(s+t \eta), \frac{n+t \zeta}{|n+t \zeta|}, \nabla\left(\frac{n+t \zeta}{|n+t \zeta|}\right)\right) d x \\
=\int_{\Omega}\left[\tilde{W}_{p_{i}^{k}}\left[\frac{\partial \zeta^{k}}{\partial x_{i}}-\frac{\partial}{\partial x_{i}}\left(n^{k} n^{j} \zeta^{j}\right)\right]+\tilde{W}_{s_{i}} \frac{\partial \eta}{\partial x_{i}}\right. \\
\left.+\tilde{W}_{u^{k}}\left(\zeta^{k}-n^{k} n^{j} \zeta^{j}\right)+\tilde{W}_{s} \eta\right] d x
\end{gathered}
$$

for $\zeta \in H_{0}^{1}\left(\Omega ; \mathbb{R}^{3}\right) \cap L^{\infty}$, and $\eta \in H_{0}^{1}(\Omega ; \mathbb{R}) \cap L^{\infty}$, we have

$$
\left\{\begin{array}{c}
-\frac{\partial}{\partial x_{i}} \tilde{W}_{p_{i}^{k}}+n^{k} n^{j} \frac{\partial}{\partial x_{i}} \tilde{W}_{p_{i}^{j}}+\tilde{W}_{u^{k}}-n^{k} n^{j} \tilde{W}_{u^{j}}=0 \\
k=1,2,3 \\
-\frac{\partial}{\partial x_{i}} \tilde{W}_{s_{i}}+\tilde{W}_{s}=0
\end{array}\right.
$$

where $s_{i}=\frac{\partial s}{\partial x_{i}}$ for $i=1,2,3$. 
To derive a Pohozaev's identity, for any $\bar{x} \in \Omega$ and $\bar{s} \in\left(-\frac{1}{2}, 1\right)$, we compute

$$
\begin{aligned}
\frac{\partial}{\partial x_{i}}[ & \left.\left(x_{i}-\bar{x}_{i}\right)\left(\tilde{W}-W_{0}(\bar{s})\right)-\left(x_{j}-\bar{x}_{j}\right) \frac{\partial n^{k}}{\partial x_{j}} \tilde{W}_{p_{i}^{k}}-\left(x_{j}-\bar{x}_{j}\right) \frac{\partial s}{\partial x_{j}} \tilde{W}_{s_{i}}\right] \\
= & 3\left(\tilde{W}-W_{0}(\bar{s})\right)+\left(x_{i}-\bar{x}_{i}\right) \tilde{W}_{p_{j}^{k}} \frac{\partial^{2} n^{k}}{\partial x_{i} \partial x_{j}}+\left(x_{i}-\bar{x}_{i}\right) \frac{\partial^{2} s}{\partial x_{i} \partial x_{j}} \tilde{W}_{s_{j}} \\
& +\left(x_{i}-\bar{x}_{i}\right) \tilde{W}_{u^{k}} \frac{\partial n^{k}}{\partial x_{i}}+\left(x_{i}-\bar{x}_{i}\right) \tilde{W}_{s} \frac{\partial s}{\partial x_{i}}-\frac{\partial n^{k}}{\partial x_{i}} \tilde{W}_{p_{i}^{k}} \\
& -\left(x_{j}-\bar{x}_{j}\right) \frac{\partial^{2} n^{k}}{\partial x_{i} \partial x_{j}} \tilde{W}_{p_{i}^{k}}-\left(x_{j}-\bar{x}_{j}\right) \frac{\partial n^{k}}{\partial x_{j}} \frac{\partial}{\partial x_{i}} \tilde{W}_{p_{i}^{k}}-\frac{\partial s}{\partial x_{i}} \tilde{W}_{s_{i}} \\
& -\left(x_{j}-\bar{x}_{j}\right) \frac{\partial^{2} s}{\partial x_{i} \partial x_{j}} \tilde{W}_{s_{i}}-\left(x_{j}-\bar{x}_{j}\right) \frac{\partial s}{\partial x_{j}} \frac{\partial}{\partial x_{i}} \tilde{W}_{s_{i}} \\
= & 3\left(W_{0}(s)-W_{0}(\bar{s})\right)+s^{2} W(n, \nabla n)+k_{5}|\nabla s|^{2}+k_{6}|\nabla s \cdot n|^{2} \\
& +\left(x_{i}-\bar{x}_{i}\right) \tilde{W}_{u^{k}} \frac{\partial n^{k}}{\partial x_{i}}+\left(x_{i}-\bar{x}_{i}\right) \tilde{W}_{s} \frac{\partial s}{\partial x_{i}}-\left(x_{j}-\bar{x}_{j}\right) \frac{\partial n^{k}}{\partial x_{j}} \\
& \times\left(n^{k} n^{j} \frac{\partial}{\partial x_{i}} \tilde{W}_{p_{i}^{j}}+\tilde{W}_{u^{k}}-n^{k} n^{j} \tilde{W}_{u^{j}}\right)-\left(x_{j}-\bar{x}_{j}\right) \frac{\partial s}{\partial x_{j}} \tilde{W}_{s} \\
= & 3\left(W_{0}(s)-W_{0}(\bar{s})\right)+s^{2} W(n, \nabla n)+k_{5}|\nabla s|^{2}+k_{6}|\nabla s \cdot n|^{2} \\
& -\left(x_{j}-\bar{x}_{j}\right) \frac{\partial n^{k}}{\partial x_{j}} n^{k} n^{j}\left(\frac{\left.\partial \tilde{W}_{p_{i}^{j}}-\tilde{W}_{u^{j}}\right) .}{\partial x_{i}}\right.
\end{aligned}
$$

Then, by the fact that $|n|=1$,

$$
\begin{aligned}
& \frac{\partial}{\partial x_{i}}\left[\left(x_{i}-\bar{x}_{i}\right)\left(\tilde{W}-W_{0}(s)\right)-\left(x_{j}-\bar{x}_{j}\right) \frac{\partial n^{k}}{\partial x_{j}} \tilde{W}_{p_{i}^{k}}-\left(x_{j}-\bar{x}_{j}\right) \frac{\partial s}{\partial x_{j}} \tilde{W}_{s_{i}}\right] \\
& \quad=3\left(W_{0}(s)-W_{0}(\bar{s})\right)+s^{2} W(n, \nabla n)+k_{5}|\nabla s|^{2}+k_{6}|\nabla s \cdot n|^{2} .
\end{aligned}
$$

Now, consider $\left.(s, n)\right|_{\partial \Omega}=\left(s_{0}, n_{0}\right) \equiv$ const. so that

$$
\begin{gathered}
\int_{\partial \Omega}(x-\bar{x}) \cdot \nu\left(\tilde{W}-W_{0}(\bar{s})-\frac{\partial n^{k}}{\partial x_{i}} \tilde{W}_{p_{i}^{k}}-\frac{\partial s}{\partial x_{i}} \tilde{W}_{s_{i}}\right) d \mathcal{H}^{2} \\
=\int_{\Omega}\left[3\left(W_{0}(s)-W_{0}(\bar{s})\right)+s^{2} W(n, \nabla n)\right. \\
\left.\quad+k_{5}|\nabla s|^{2}+k_{6}|\nabla s \cdot n|^{2}\right] d x
\end{gathered}
$$


Fixing $\bar{s}=s_{0}$ and using the fact that $W$ is quadratic in $\nabla n$ and $k_{5}|\nabla s|^{2}+k_{6}|\nabla s \cdot n|^{2}$ is quadratic in $\nabla s$, by Euler's identity, it yields

$$
\begin{aligned}
(2.17)- & \int_{\partial \Omega}(x-\bar{x}) \cdot \nu\left(s^{2} W(n, \nabla n)+k_{5}|\nabla s|^{2}+k_{6}|\nabla s \cdot n|^{2}\right) d \mathcal{H}^{2} \\
= & \int_{\Omega}\left[3\left(W_{0}(s)-W_{0}\left(s_{0}\right)\right)+s^{2} W(n, \nabla n)\right. \\
& \left.\quad+k_{5}|\nabla s|^{2}+k_{6}|\nabla s \cdot n|^{2}\right] d x .
\end{aligned}
$$

Before giving a constancy result, we want to remark that the boundary value $s_{0}$ is necessary a critical point of function $W_{0}(s)$ when $(s, n)$ is a constant stationary point of (2.14). In fact, this can be easily checked the Euler-Lagrange equation (2.15).

THEOREM 3. - Assume that $\Omega$ is star-shaped and $(s, n)$ is a smooth solution for (2.15) with $\left.s\right|_{\partial \Omega}=s^{*}$, and $\left.n\right|_{\partial \Omega}=$ const. Then $(s, n)$ is constant in $\Omega$.

Proof. - The desired result follows easily by taking $s_{0}=s^{*}$, and $\bar{x} \in \Omega$ such that $\Omega$ is star-shaped with respect to $\bar{x}$ in $(2.17)$.

\section{NON-STARSHAPED DOMAINS}

To make all the subsequent discussion simpler, we shall focus on the special case (1.2) which is the integrand for a harmonic mapping from a bounded smooth domain $\Omega$ in $\mathbb{R}^{3}$ into $S^{2}$. (In fact, it is possible to replace the target $S^{2}$ by a Riemannian manifold. However, we shall not consider this general case here.) The bulk energy functional is

$$
E(n)=\int_{\Omega}|\nabla n|^{2} d x \quad \text { for } \quad n \in H^{1}\left(\Omega ; S^{2}\right)
$$

and the equilibrium state satisfies

$$
-\Delta n=n|\nabla n|^{2} \quad \text { in } \mathcal{D}^{\prime}\left(\Omega ; \mathbb{R}^{3}\right)
$$

It was shown in [3] that there exist nonconstant smooth harmonic maps from $\Omega$ into $S^{2}$ if $\partial \Omega$ is not connected. This result seems to suggest that the constancy theorems only hold for star-shaped domains. Nevertheless, in this section, we shall prove the following result: 
THEOREM 4. - There exists a non-starshaped bounded domain $\Omega$ on which any solution $n$ of (3.2) is identically equal to a constant if it is constant on $\partial \Omega$.

Let's consider a smooth solution $n$ of (3.2). Denote by $h=\left(h^{1}, h^{2}, h^{3}\right)$ a smooth vector field on $\bar{\Omega}$. Following [12], we compute, by (3.2),

$$
\begin{aligned}
\frac{\partial}{\partial x_{i}} & {\left[h^{i}|\nabla n|^{2}-h^{j} \frac{\partial n^{k}}{\partial x_{j}}\left(2 \frac{\partial n^{k}}{\partial x_{i}}\right)\right] } \\
= & \frac{\partial h^{i}}{\partial x_{i}}|\nabla n|^{2}+2 h^{i} \frac{\partial n^{k}}{\partial x_{j}} \frac{\partial^{2} n^{k}}{\partial x_{i} \partial x_{j}}-2 \frac{\partial h^{j}}{\partial x_{i}} \frac{\partial n^{k}}{\partial x_{j}} \frac{\partial n^{k}}{\partial x_{i}} \\
& -h^{j} \frac{\partial^{2} n^{k}}{\partial x_{i} \partial x_{j}}\left(2 \frac{\partial n^{k}}{\partial x_{i}}\right)-h^{j} \frac{\partial n^{k}}{\partial x_{j}}\left(2 \Delta n^{k}\right), \\
= & \frac{\partial h^{i}}{\partial x_{i}}|\nabla n|^{2}-2 \frac{\partial h^{j}}{\partial x_{i}} \frac{\partial n^{k}}{\partial x_{j}} \frac{\partial n^{k}}{\partial x_{i}}+h^{j} \frac{\partial n^{k}}{\partial x_{j}} n^{k}|\nabla n|^{2} .
\end{aligned}
$$

As $|n|=1$, it follows that

$$
\frac{\partial}{\partial x_{i}}\left(h^{i}|\nabla n|^{2}-2 h^{j} \frac{\partial n^{k}}{\partial x_{i}} \frac{\partial n^{k}}{\partial x_{j}}\right)=\frac{\partial h^{i}}{\partial x_{i}}|\nabla n|^{2}-2 \frac{\partial h^{j}}{\partial x_{i}} \frac{\partial n^{k}}{\partial x_{i}} \frac{\partial n^{k}}{\partial x_{j}} .
$$

Now, if we assume $n=$ const. on $\partial \Omega$, then

$$
h^{j} \frac{\partial n^{k}}{\partial x_{i}} \frac{\partial n^{k}}{\partial x_{j}} \nu^{i}=|\nabla n|^{2} h \cdot \nu \quad \text { on } \partial \Omega .
$$

As in Section 2, applying the divergence theorem, we get

$$
-\int_{\partial \Omega}|\nabla n|^{2} h \cdot \nu d \mathcal{H}^{2}=\int_{\Omega}\left(\frac{\partial h^{i}}{\partial x_{i}}|\nabla n|^{2}-2 \frac{\partial h^{i}}{\partial x_{i}} \frac{\partial n^{k}}{\partial x_{i}} \frac{\partial n^{k}}{\partial x_{j}}\right) d x
$$

The conformal group of the Euclidean space is spanned by translations, rotations, dilatations and inversions. So far we have only used the translations and dilatations. They yield constancy theorems on star-shaped domains respectively. Now let's look at the inversions, $\xi^{k}, k=1,2,3$,

$$
\left\{\begin{array}{l}
\xi_{k}^{k}=x_{k}^{2}-\sum_{i \neq k} x_{i}^{2} \\
\xi_{j}^{k}=2 x_{k} x_{j}, \quad j=1,2,3, \quad j \neq k .
\end{array}\right.
$$

Let's just consider $\xi^{1}$. Setting $h=\xi^{1}$ in (3.3), we get

$$
-\int_{\partial \Omega}|\nabla n|^{2} \xi^{1} \cdot \nu d \mathcal{H}^{2}=2 \int_{\Omega} x_{1}|\nabla n|^{2} d x
$$


Therefore, if we can find a domain $\Omega$ contained in $\left\{x: x_{1} \geq 0\right\}$ along whose boundary $\xi^{1} \cdot \nu \geq 0$, we get a constancy result.

Such a domain can be constructed as follows. Let's consider the system of differential equations

$$
\left\{\begin{array}{l}
\frac{d x_{1}}{d t}=x_{1}^{2}-x_{2}^{2} \\
\frac{d x_{2}}{d t}=2 x_{1} x_{2}
\end{array}\right.
$$

in $\left(x_{1}, x_{2}\right)$-plane. A first integral of this system is given by

$$
x_{2}\left(x_{1}\right)=\left[1-\left(1-4 k^{2} x_{1}^{2}\right)^{1 / 2}\right] / 2 k
$$

where $k$ is a parameter; when $k \rightarrow 0^{+}, x_{2}(1) \rightarrow 0$, and when $k \rightarrow \frac{1}{2}$, $x_{2}(1) \rightarrow 1$. Each integral curve hits the diagonal $x_{1}=x_{2}$ vertically at $(1 / 2 k, 1 / 2 k)$.

Let's fix four constants: $k \in(0,1 / 2), R \in(1 / 2 k, \infty), \alpha \in(0,1)$, and $\beta \in(1 / 2 k, R)$. Consider an arc and two line segments in the $\left(x_{1}, x_{2}\right)$-plane,

$$
\begin{aligned}
\overline{A B}= & \left\{\left(x_{1}, x_{2}\right) \in \mathbb{R}^{2} ; x_{1} \in[0,1], x_{2}=\alpha\right\} \\
\overline{C D}= & \left\{\left(x_{1}, x_{2}\right) \in \mathbb{R}^{2} ; x_{1} \in[1,1 / 2 k],\right. \\
& \left.x_{2}=\left[1-\left(1-4 k^{2} x_{1}^{2}\right)^{1 / 2}\right] / 2 k .\right\} \\
\overline{E F}= & \left\{\left(x_{1}, x_{2}\right) \in \mathbb{R}^{2} ; x_{1} \in[1 / 2 k, R], x_{2}=\beta .\right\}
\end{aligned}
$$

Connect $B$ with $C$ and $D$ with $E$ by straight line segments. Together with the $x_{1}$-axis, and the two vertical lines (through points $A$ and $F$ respectively), this curve bounds a domain $\omega$ in the $\left(x_{1}, x_{2}\right)$-plane. See the figure below.

Now we smooth out the corners at $A, B, C, E, F$ to get a domain $\omega^{\prime}$ such that

(i) $\partial \omega^{\prime}$ contains a straight line segment in $\overline{B C}$ and a straight line segment in $\overline{D E}$;

(ii) The outer normal $\nu=\left(\nu^{1}, \nu^{2}\right)$ at the curves which smooth out the corners $A, E$ satisfies $\nu^{1} \leq 0, \nu^{2} \geq 0$;

(iii) The outer normal $\nu=\left(\nu^{1}, \nu^{2}\right)$ at the curves which smooth out the corners $B, F$ satisfies $\nu^{1} \geq 0, \nu^{2} \geq 0$;

(iv) The outer normal $\nu=\left(\nu^{1}, \nu^{2}\right)$ at the curves which smooth out the corner $C$ satisfies $\left(x_{1}^{2}-x_{2}^{2}\right) \nu^{1}+2 x_{1} x_{2} \nu^{2} \geq 0$. Then one easily checks that

$$
\left(x_{1}^{2}-x_{2}^{2}\right) \nu^{1}+2 x_{1} x_{2} \nu^{2} \geq 0 \quad \text { on } \partial \omega^{\prime} \backslash x_{1} \text {-axis. }
$$




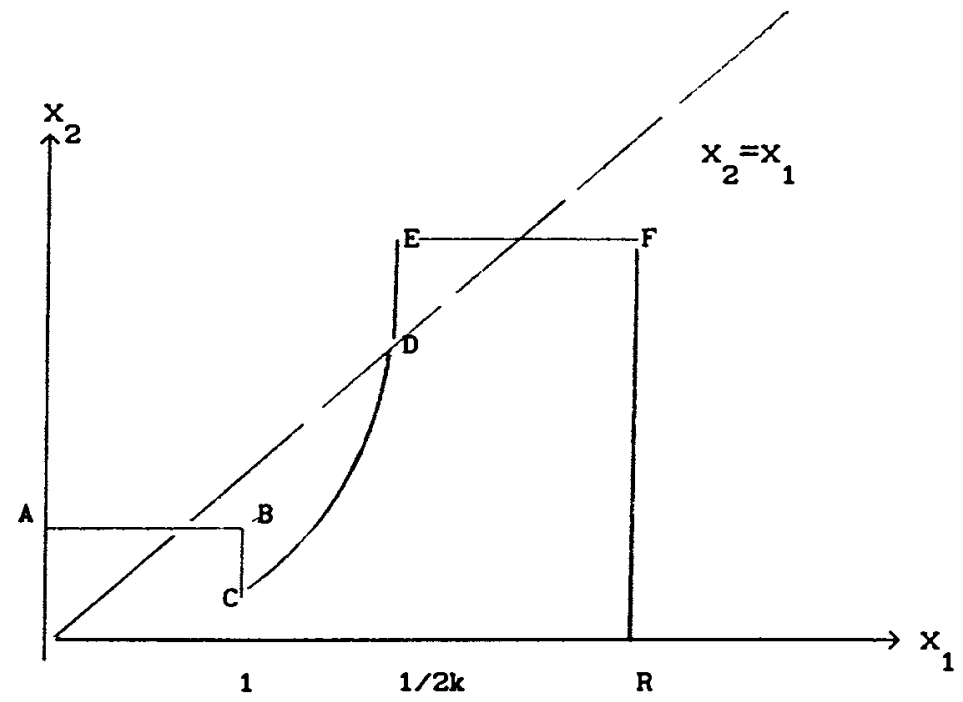

We rotate the domain $\omega^{\prime}$ around the $x_{1}$-axis in $\mathbb{R}^{3}$ to get a smooth domain $\Omega$ in $\mathbb{R}^{3}$ which satisfies $\Omega \subseteq\left\{x \in \mathbb{R}^{3}: x_{1} \geq 0\right\}$ and

$$
\left(x_{1}^{2}-x_{2}^{2}-x_{3}^{2}\right) \nu^{1}+2 x_{1} x_{2} \nu^{2}+2 x_{1} x_{3} \nu^{3} \geq 0,
$$

i.e., $\xi^{1} \cdot \nu \geq 0$ on $\partial \Omega$.

Moreover, from (i) above, we know that the domain $\Omega$ is non-starshaped. Hence we conclude that Theorem 4 holds.

Remark 1. - Many non-starshaped domains with similar property can be constructed. For instance, fix a point $C^{\prime}$ on the line segment $\overline{F R}$ and draw $C^{\prime} D^{\prime} E^{\prime} F^{\prime} R^{\prime}$ like $C D E F R$ where $\overline{C^{\prime} D^{\prime}}$ is also a portion of an integral curve of (3.4). Then $A B C D E F C^{\prime} D^{\prime} E^{\prime} F^{\prime} R^{\prime}$ forms a new $\omega$. After smoothing and rotating $\omega$ we get a new non-starshaped domain over which constancy results hold. In fact by taking a point $C^{\prime \prime}$ on $\overline{F^{\prime} R^{\prime}}$ we may again form another $\omega$. By doing this repeatedly we obtain many such non-starshaped domains.

Remark 2. - Our construction also yields a non-starshaped domain in $\mathbb{R}^{n}$, $n \geq 3$, such that $\xi^{1} \cdot \nu \geq 0$ along its boundary where $\xi^{1}$ is the inversion along the $x_{1}$-direction: $\xi_{1}^{1}=x_{1}^{2}-\sum_{j>1} x_{j}^{2}$ and $\xi_{i}^{1}=2 x_{1} x_{i}, i=2, \ldots, n$. One simply has to rotate the domain $\omega^{\prime}$ around $\mathbb{R}^{n-1}$. 
Now we turn to the Dirichlet problem (1.4). The equation therein is the Euler-Lagrange equation for the Lagrangian

$$
\mathcal{F}=\frac{1}{2}|\nabla u|^{2}-\frac{u^{p+1}}{p+1}
$$

Let $u$ be a $C^{2}$-solution of (1.4). According to [12] the following identity holds:

$$
\begin{aligned}
& \frac{\partial}{\partial x_{i}}\left(h^{i} \mathcal{F}-h^{j} u_{j} \mathcal{F}_{p_{i}}-a u \mathcal{F}_{p_{i}}\right) \\
& \quad=\frac{\partial h^{i}}{\partial x_{i}} \mathcal{F}-\left(u_{j} \frac{\partial h^{j}}{\partial x_{i}}+u \frac{\partial a}{\partial x_{i}}\right) \mathcal{F}_{p_{i}}-a\left(u_{i} \mathcal{F}_{p_{i}}+u \mathcal{F}_{u}\right),
\end{aligned}
$$

where $h$ is a vector field and $a$ is a smooth function. We claim that no positive solution can exist in a domain as described in Remark 2 . For, take $h$ to be $\xi^{1}$ and $a$ to be a linear function $\lambda x_{1}$ in this identity and then integrate it over $\Omega$. By the divergence theorem we have

$$
\begin{aligned}
\int_{\partial \Omega} & \left(\frac{1}{2} \xi^{1} \cdot \nu|\nabla u|^{2}-\xi_{j}^{1} \frac{\partial u}{\partial x_{j}} \frac{\partial u}{\partial x_{i}} \nu^{i}\right) \\
= & \int_{\Omega}\left[(n-2-\lambda) x_{1}|\nabla u|^{2}+\left(\lambda-\frac{2 n}{p+1}\right) x_{1} u^{p+1}-\lambda u \frac{\partial u}{\partial x_{1}}\right]
\end{aligned}
$$

By the fact that $u$ vanishes on the boundary on $\Omega$, we get

$$
\int_{\Omega} \lambda u \frac{\partial u}{\partial x_{1}}=\frac{\lambda}{2} \int_{\Omega} \frac{\partial u^{2}}{\partial x_{1}}=0
$$

and, for $j=1,2, \ldots, n$,

$$
\frac{\partial u}{\partial x_{j}}=\frac{\partial u}{\partial \nu} \nu^{i} \text { on } \partial \Omega
$$

So

$$
\xi_{j}^{1} \frac{\partial u}{\partial x_{j}} \frac{\partial u}{\partial x_{i}} \nu^{i}=\xi_{j}^{1} \nu^{j} \frac{\partial u}{\partial \nu} \frac{\partial u}{\partial x_{i}} \nu^{i}=\xi^{1} \cdot \nu|\nabla u|^{2} \quad \text { on } \partial \Omega .
$$

Thus, we deduce the following Pohozaev's identity:

$-\frac{1}{2} \int_{\partial \Omega} \xi^{1} \cdot \nu|\nabla u|^{2}=\int_{\Omega}(n-2-\lambda) x_{1}|\nabla u|^{2}+\left(\lambda-\frac{2 n}{p+1}\right) x_{1} u^{p+1}$.

The second term on the right hand side of this identity can be dropped by choosing $\lambda=2 n /(p+1)$. It follows that no positive solution can exist when $p \geq(n+2) /(n-2)$. In conclusion we have established 
THEOREM 5. - There exists a non-starshaped bounded domain $\Omega$ over which (1.4) has no solution.

\section{ACKNOWLEDGEMENTS}

The first author was partially supported by the Earmarked Grant for Research (Hong Kong). The second author wishes to thank the Department of Mathematics of the Chinese University of Hong Kong for its hospitality during the period when this work was carried out, and also the Youth Foundation, NSFC, for its partial support. Both authors would like to thank the referee for his constructive comments on the manuscript.

\section{REFERENCES}

[1] A. BAHRI and J. M. CoRon, On a nonlinear elliptic problem involving the critical Sobolev exponent: The effect of the topology of the domain, Com. Pure Appl. Math., Vol. 41, 1988, pp. 253-294.

[2] F. BETHuEL and H. BREzis, Regularity of minimizers of relaxed energies for harmonic maps, C. R. Acad. Sci. Paris, t. 310, Series I, 1990, pp. 827-829.

[3] F. Bethuel, H. Brezis and J. M. Coron, Relaxed energies for harmonic maps, in Variational Problems, H. Berestycki, J. M. CORON and I. EKELAND eds., Birkhäuser, 1990.

[4] W. DING, Positive solutions of $\Delta u+u^{(n+2) /(n-2)}=0$ on a contractible domain, preprint.

[5] J. L. ERICKSEN, Equilibrium theory of liquid crystals, in Advances in Liquid Crystals, 2 , pp. 233-299, G. H. Brown ed., New York: Academic Press, 1976.

[6] R. HARDT and D. KINDERLEHRER, Mathematical questions of liquid crystal theory, in Theory and Applications of Liquid Crystals, IMA, 5, Springer-Verlag, 1986.

[7] R. HARDT, D. KinderLeHRER and F. H. Lin, Existence and partial regularity of static liquid crystal configurations, Com. Math. Phy., Vol. 105, 1986, pp. 547-570.

[8] H. KARChER and J. C. WoOD, Non-existence results and growth properties for harmonic maps and forms, J. Reine Angew. Math., Vol. 353, 1984, pp. 165-180.

[9] L. LEMAIRE, Applications harmoniques de surfaces riemanniennes, J. Diff. Geom., Vol. 13, 1978, pp. 51-78.

[10] F. H. LiN, Nonlinear theory of defects in nematic liquid crystal; phase transition and flow phenomena, Com. Pure. Appl. Math., Vol. 42, 1989, pp. 789-814.

[11] S. I. PohozaEv, Eigenfunction of the equation $\Delta u+\lambda f(u)=0$, Soviet Math. Dokl., Vol. 6, 1965, pp. 1408-1411.

[12] P. Pucci and J. Serrin, A general variational identity, Indiana Univ. Math. J., Vol. 35, 1986, pp. 681-703.

[13] J. C. Wood, Non-existence of solutions to certain Dirichlet problems for harmonic maps, preprint, Leeds Univ., 1981.

[14] J. Eells and L. Lemaire, A report on harmonic maps, Bull. London Math. Soc., Vol. 10, 1978, pp. 1-68. 